\title{
Intelligent Algorithms of Wireless Communication for Internet of Things Service Security and Privacy Assurance
}

\section{Yuanguo Wang}

College of Information Engineering, Binzhou Polytechnic

Xiaogang Jiang

College of Information Engineering, Binzhou Polytechnic

Qian Yu (D yq_2165@126.com )

Binzhou Polytechnic

\section{Xiuling Zhang}

College of Information Engineering, Binzhou Polytechnic

\section{Bailu Zhao}

College of Information Engineering, Binzhou Polytechnic

Xiaobei Wang

College of Information Engineering, Binzhou Polytechnic

Tonghui Han

College of Information Engineering, Binzhou Polytechnic

\section{Research Article}

Keywords: Internet of Thing, service security, privacy protection, collaborative interaction

Posted Date: July 28th, 2021

DOI: https://doi.org/10.21203/rs.3.rs-749325/v1

License: (c) (1) This work is licensed under a Creative Commons Attribution 4.0 International License. Read Full License 


\section{Abstract}

Due to its huge application potential, the Internet of Things has received extensive attention from governments, academia and industry. The core concepts of the Internet of Things are perception, control, transmission and intelligence. Through technical means, the coordination of things and things, people and things, and people and people has been realized, thus forming a network based on sensor networks, the Internet, and mobile communication networks. A larger complex network system. However, restricted by the characteristics of network structure, terminal equipment, communication methods, application scenarios, etc., some security and privacy issues unique to the Internet of Things cannot be directly solved by existing Internet security technologies. Aiming at the general high complexity of existing algorithms, this article starts with the different phase-frequency characteristics of different filters, and designs a new low-complexity reduction system algorithm. According to the characteristics of the system that the filter structure can be flexibly selected, the method randomly allocates different filters to each sub-carrier and adjusts the phase of signal superimposition, thereby constructing a coordinated communication facility and management service coordination suitable for large-scale distributed loT services. The interactive access control architecture realizes the confidentiality of data exchange between services.

\section{Introduction}

Facing various physical goals in the real world, smart terminals help loT systems analyze, collect, analyze, and process information and data in a timely or delayed manner [1-3]. The Internet of Things system finally completes "perception and communication" with various physical targets in the real world by connecting wired/wireless network systems. Generally speaking, the Internet of Things system can be considered as a kind of "Internet", used for wide area network/local area network, identification of information and data, and communication between individuals and targets to be identified [4]. At present, there are still many immature parts in the technology of the Internet of Things system. In the Internet of Things, data information is generally not encrypted or transmitted directly. Various types of sensors and network systems are at risk of being invaded by potential intruders [5-8]. Therefore, it is of great practical significance to analyze and research the Internet of Things system from the perspective of system security and promote the gradual improvement of the Internet of Things system [9].

At the same time, the security issues of the Internet of Things system in the intelligent industrial control system have also surfaced. At this stage, with the development of the Industrial Internet of Things, IT and OT are gradually converging [10-14]. As an important part of the "Industry 4.0" project, loT systems, production equipment, industrial software systems and production control systems are interconnected through the Industrial Internet of Things. This situation makes it difficult to detect security threats to the Internet of Things system, and it is even more difficult to troubleshoot and repair the security risks of the Internet of Things system [15]. The loT platform needs to have a high degree of flexibility and sensitivity in order to accurately deploy the appropriate technology, spectrum and bandwidth according to the requirements of each application, and support the efficient multiplexing of future-oriented services and 
terminal types. The advantage of the intelligent algorithm is that the pulse shaping filter can be flexibly selected to filter the sub-carrier signal, which inspired us to propose a new suppression algorithm that uses different filters for different sub-carriers to improve the peak-to-average ratio distribution.

The Internet is the foundation of loT construction, so the security threat of the loT includes two aspects. First of all, the security problems encountered in the Internet will appear in the loT; secondly, the loT system itself and related applications are also vulnerable. At present, the status quo of China's network security is not optimistic [16-18]. First, in the public Internet environment, the traits of hacker attacks are becoming more and more obvious. The criminals aim at communication networks, information systems, and user information and property. They use hacker technology to launch cyber attacks to gain illegal interests, and gradually form a tight organization and division of labor. Clear Internet underground industry [19-21].The security of the loT system itself is mainly reflected in the perception level of the loT. In the overall architecture of the loT, the perception level is at the lowest and most basic level. This level of information security is the most vulnerable.The sensing layer mainly uses RFID and WSNs in the process of collecting information. The security of the IOT perception layer is essentially a security issue for RFID systems and WSNs systems [22]. When sensors receive information through a wireless sensor network, a large number of sensor nodes are needed. These nodes are often exposed in public places, controlled remotely by individuals or computers, lack effective protection, and are sensitive to signal interference and even interference from node capture. Furthermore, there are many security vulnerabilities in the routing protocols of WSNs, and criminals can inject malicious routing information into WSNs to make the network paralyzed. With the rapid application and application of RFID technology, its data security problem has even exceeded [23-24]. The main reasons are: weak computing power of the card; the vulnerability of the wireless network causes the transmitted information to be exposed to the public; the privacy and security of commercial applications make the security and privacy of the transmitted information an important factor, which limits the further development of the loT.

In view of the characteristics of loT services and the above security and privacy challenges, this paper has conducted in-depth research on the key issues of security and privacy protection technologies for services in the loT environment. Researching highly scalable data access control, services and user privacy protection mechanisms provides theoretical guidance and practical value for building secure loT services.

\section{Proposed Method}

\subsection{Privacy protection}

Privacy is a concept formed by human society in the context of the concept of private ownership in the civilized era. Privacy is defined differently for different domains and objects. There is no clear definition of a more general and broad concept of privacy. This article believes that for personal or collective security and interests, the information that is closely related to itself is hidden to prevent outsiders from 
knowing that such hidden information is "privacy." Privacy information is information that individuals, groups, and other entities are reluctant to be aware of by the outside world. The act taken to achieve covert privacy information is called "confidentiality of privacy protection", that is, "privacy protection."

In order to provide users with ubiquitous personalized services, the loT must use automated perception functions to use users' personal information without being perceived or disturbed by users. For example, in the intelligent medical care system, it is necessary to collect physiological physiological data (heart rate, body temperature, blood pressure, etc.) of the user in real time. In the loT environment, the use of personal information covers the entire life cycle of users' personal data, including its perception, storage, transmission and application. User privacy issues mainly occur in the sensing and application phases of these four processes. Data perception has the characteristics of invisible and wide coverage, and belongs to system behavior. Because perceived personal information is private to users, users have privacy protection requirements for this process. In addition, service-oriented application processing the essence is that the personal information is shared by other entities interacting with the system. For individuals, the information is uncontrollable, and the user also needs privacy protection for this process. In these cases, if the system privacy protection mechanism is missing, the user's private information will be potentially threatened, which raises the privacy protection of the loT. As a special concept, privacy protection has the timeliness and mandatory characteristics of time and space in the historical process of each entity. Although it is for unauthorised persons, privacy protection has its special significance compared with the privacy of information security in general. Privacy can be considered as the privacy of certain information, and unlike general information privacy, in many cases, privacy and external needs must be balanced. For example, when a doctor provides a diagnosis service to a patient, some patients may be ashamed to disclose their personal information, but have to provide their doctor with their own historical medical records. The source of balance between external demand and privacy protection is often due to political or other social coercive factors. In addition, privacy protection has a broader, relatively independent system and variability for different spaces and times. Therefore, the goal of privacy protection technology is not to completely hide private information, but to meet the certain information needs of the outside world while leaking as little as possible, that is, to balance external needs and privacy protection. Privacy protection technology refers to the general term for all technologies that can be used to protect privacy.

\subsection{Strategy Synthesis Research for Secure Data Sharing}

In order to provide better services to users, different organizations need to cooperate with each other. The main goal of this collaboration is data sharing. Data shared in a service collaborative environment is generally sensitive, such as medical data for patients in a medical information system. Therefore, providing security guarantees for sharing data is an urgent problem to be solved. To meet this challenge, a common global strategy for any participating organization that all participating organizations can accept. Working with a global strategy usually requires a coordinated or negotiated approach between organizations. An example of a combination of services is the production of policy-based services, which requires a policy integrating each component service. Therefore, the key to defining a strategy controlling 
access to shared data is a combination of local policies of different participating organizations into one global strategy. Generally, there are two layers of strategy for la joint data for data in the format 728212; the layer is a coarse data layer, organizational layer, data can be files, databases or other information; the second layer is the data layer associated with the data structure. Research in this section focuses on the coarse data layer. To protect your data, different organizations usually choose different attribute elements and restricting access to control to independently dictate the management policy of data usage, which can easily lead to misunderstandings between organizations. Strategies of different organizations are different, even dissuasive, such as one rule allows some action on common data, and the second rule does not allow the same operation on shared data. Therefore, the key issue in the combination of strategy is standardization and integration of policy principles, a way of solving various and even rejected principles. To solve the above problem, the first task is to standardize in Demographic language plays an important role in regulating these requirements. Different policy languages have appeared, such as XACL, EPAL and XACML, which have provided algorithms for the combination of strategies. But mainly focus on pre-ionic algorithm combinations of policies such as enabling priority, rejection of priority and so on. These methods are not enough to support the complex semantics of a data-driven policy combination. This article will use this strategy to combine the principles to combine the strategy.

XACML is one of the most commonly used policy specification languages. If the loT system runs with fewer communication ports, it will cause a series of security risks if the carrying capacity of the network system exceeds its personal carrying capacity. The specific manifestations are as follows: First, network system congestion. The operation of the whole network system includes a large number of network devices, and the current authentication analysis method can not effectively manage the use of each device. In this case, how to ensure the connection between most multiplier devices and network systems becomes a problem that relevant personnel need to think about and solve. Second, Computer communication network terminals adopt a unified authentication method to encrypt and manage various information. In this process, if there are other devices connected to the loT, a lot of resources will be wasted when authentication generates secret keys. Thirdly, system transmission security. Computer communication network can acquire more complete information under the action of encryption algorithm, but due to the interference of network equipment, if the encryption algorithm is more complex, it will lead to the problem of information use delay. IoT (IOT) is essentially a technology that integrates and superimposes data information through radio frequency identification (RFID), infrared sensing and global positioning system (GPS). It promotes passive recipients to scan and track the used items, and transforms the privacy data information into public information. However, due to the lack of effective protection of information, the whole system will lose information when it is used. 2.3 loT terminal node security issues loT equipment will generally be arranged in various unmanned monitoring scenarios, in the geographical space distribution reflects the characteristics of decentralization, which brings adverse effects for attackers attacking system equipment, seriously interfering with the safe use of the entire system equipment software and hardware. When these devices are used, they are often in a state of unmanned adherence and random operation, which invisibly increases the risk of equipment damage. loT terminal signal interference security issues. The loT perception layer network is mainly in the form of 
wireless connection. The use of signals has a strong openness, so the signal is very vulnerable to interference from the external environment when it is used. Data transmission security. Broadcasting is a main way of data transmission in the perception layer. However, in practical application, the ability of perception nodes has limitations, which increases the possibility of data information being destroyed invisibly. In addition, the perceptual nodes in the perceptual layer do not have complete data processing functions, and data errors are inevitable in the process of data use, which restricts the security and stability of the data information of the whole system. Compared with traditional network devices, intelligent sensor terminal devices generally have the characteristics of openness and comprehensiveness. In the process of using information, it is easy to be exposed to the attacker's line of sight, which provides an opportunity for attackers to attack the system and poses a threat to the cooperative work of sensor nodes.

The network system carries out isolation processing, and builds system access control with higher security level when the whole system is running, so as to realize effective isolation of different network systems. Intrusion detection technology in the application layer of the loT can help the relevant personnel to detect and detect the phenomenon of intrusion in time, and choose effective measures to repair system vulnerabilities according to the basic performance of intrusion. The computer network security based on the loT is an important link in the operation of the entire loT system, and also an important guarantee to ensure data information security. In the new historical period, combined with the actual development of the loT technology, relevant personnel need to take effective measures to protect the data security of the loT system from the use needs of the whole network, so as to better promote the development of the loT, benefit mankind and society.

\subsection{Service Access Control Based on Publish / Subscribe System}

In the first structure, it is defined which entities are in a given group and a unique identifier is assigned to a given group. In the second structure is a method of exchanging data. You can specify a group as "on" or "closed" to indicate that the data source is "yes" or "not" a member of a propagation group. In the past few decades, multicast technology has been an active topic for researchers to continue to pay attention to. Traditional multicast protocols can be divided into two broad categories: 1) transport layer multicast and 2) application layer multicast. As the name implies, the TLM protocol implements multipoint data transmission. Group members copy messages to different outgoing links. By adopting an overlay structure, ALM can avoid application deployment issues while eliminating the impact on the scalability of IP multicast. However, ALM is less efficient than TLM. The communication architecture of Fig. 1 can deliver real-time data that distinguishes QoS.

The publish-subscribe service delivers messages to destinations that are interested in messages in a data-centric manner. In this process, the specific addresses of these destinations are not specified, but based on the attributes of the messages, in the form of anonymous interactions. This means that neither the publisher nor the subscriber know their identity in the system. This form of service is an event-based 
distributed system. Initially, these systems consisted only of services provided by mature, such as event services provided by the Common Object Request Broker architecture or Web service notifications that introduced event-based communication in Web services. Subsequently, DEBS evolved into a suitable middleware structure, such as an object management group, or an advanced message queue protocol, but it is usually named with a "service". It is called the notification service. Implicit calls are used to achieve strong decoupling between applications when an event occurs. This pattern allows the publisher/subscriber pattern to be formalized into a new type of interaction model. This paper proposes a data-centric access control architecture (DCACF), as shown in Fig. 2, which supports access control and in-network data integration in smart grid services.

In the loT environment, the publish and subscribe system is designed to handle large-scale interactive information and events. In a typical publish and subscribe system, it is generally composed of a publisher, a subscriber, and a system agent. The publisher and subscriber belong to the same user, and they all generate notification events containing their own set of attributes for subsequent system processing. In a publish-subscribe system, publishers provide services and data in the system or publish advertising information; subscribers seek targeted services and data for their own use in the system; system agents act as intermediaries for publishers and subscribers. Matching services are provided to users who meet the needs of both publishers.

The peak-to-average power ratio of a signal is usually used as an evaluation parameter to measure the relationship between linearity and power efficiency. The larger the value, the higher the design cost of the linear power amplifier for the mobile terminal. The wireless communication intelligent algorithm uses the linear matrix transformation distribution characteristics to provide a signal suppression scheme based on the precoding matrix, which not only effectively improves the performance of the Internet of Things service, but also has a low complexity and has no major impact on the signal spectrum.

In the publication subscription system mentioned in this chapter, system agents are usually deployed in distributed loT nodes to provide users with more convenient and rapid service. At the same time, the program also provides user access control functions to ensure system security and data privacy. Malicious users may participate in the publishing subscription system and launch attacks during packet transmission and service access. This chapter defines two types of attacks that may exist for publishing subscription systems: privacy breach attacks and illegal access attacks. (1) Privacy Disclosure Attacks In the distribution subscription system, there may be some users who collect and analyze other users' preferences and private profiles due to curiosity. A privacy breach attack attempts to acquire and disclose a user's privately sensitive information when matching related users, storing data (eg, when caching data sets), and packet transmission. (2) Illegal Access Attack In addition, a malicious user may pretend to be an authorized user to access the data or service of the target user, resulting in illegal access, access to services or data. Malicious users may even deliberately provide incorrect services and data to legitimate users, causing the publishing subscription system to function incorrectly and fail. Or refusal to pay for the use of services or data. Both types of attacks result in a large amount of communication, computing, and storage overhead, and can also cause the confidentiality and availability of the publish and subscribe 
system to be compromised. The DCACF model of the proposed scheme is similar to the commonly used honest but curious model, and the communication channel is an unsecure channel. This assumption is more in line with the actual application. All entities in the scenario (such as publishers, subscribers, and system agents) have limited computing power, with system agents having more computing and storage capabilities than publishers and subscribers. In the scenario, publishers and subscribers do not trust each other, but they all trust system agents. System agents do not trust any publishers or subscribers. In addition, all entities are honest but curious and run their system functions in strict accordance with the designed protocol. It should be noted that in the PACS mechanism mentioned in this chapter, the system agent in the publish and subscribe system is defined as safe and reliable, regardless of its corruption.

\section{Experiments}

This experiment uses a fully homomorphic encryption mechanism, which can effectively encrypt large integers. If the public mode $\mathrm{g}=100000000000031$ is selected as the parameter, the plaintext in $\mathrm{Z} 9$ can be as large as $10 \mathrm{M}$. We extended Apache ActiveMQ, a JMS middleware. The extension method is to construct a homomorphic operation on the publisher, build a key translation on a secure intermediate proxy, and build a homomorphic decryption on the subscriber. In the first step, the publisher encrypts the events that need to be published, the intermediate agent provides a release filter that publishes the event, and the key converts the encrypted event. The intermediate agent provides the subscriber's filter, which is the authorization policy of the subscription service. The subscriber decrypts the ciphertext converted by the conversion key. This experiment was conducted in a distributed test environment. Since it is difficult to estimate the performance in the publish/subscribe system without the same synchronous clock, the subscriber and the publisher are run on the same server. The server is configured as 8.0G RAM, Intel 1 Windows 7, 64 operating system. The intermediate agent runs on other servers. The server configuration is 4.0G RAM, Intel Windows 7 operating system. The latter mainly consists of three parts of delay: (1) the time of the publisher's encryption event, and (2) the time of the intermediate agent's key conversion, that is, the intermediate agent receives the encrypted event, and sends the converted re-encryption event. Time, (3) the time at which the recipient decrypts the re-encryption event. Through testing, we evaluated two aspects: one is the impact of the size of the publisher's published packet on performance, and the other is the performance impact of the number of attribute connections in the authorization policy.

\section{Discussion}

Each test case has been run 1000 times to ensure the accuracy of the test results. Figure 3 shows when you are not joining the access control architecture (Pub-to-Sub With Plain) and join the access control architecture (Pub-to-Sub with When the ACF), to publish data from the policy average delay (in number of the received data in different rules: $\mathrm{ms}$ ), the size of the data is fixed (256 Bytes). The horizontal coordinate indicates the size of the rule in the linear range of $I$, that is, $2,4,6,8,10$, and the vertical coordinate indicates the delay.

This shows that the access control architecture proposed in this paper has a good scalability in the number of attribute connections in the policy. An event, different packet sizes (from $1 \mathrm{~KB}$ to $5 \mathrm{~KB}$ ), 
estimated performance shown in Fig. 4. With the size distribution data variation can be observed in the original publish / subscribe system and added to the access control architecture overall delay (ACF) publish / subscribe system, the delay changes linearly, and it changes slowly. This means that the access control architecture proposed in this paper can support arbitrarily large data.

As can be seen from the above estimated results of delay, the proposed DCA CF architecture expanded the size of the size of the encrypted data, encryption can support large data. The publish / subscribe model is suitable for building a communication architecture for collaborative smart grid services. In Section Qi in the publish / subscribe service proposed smart grid data-centric access control architecture that maintains the publish / subscribe paradigm of anonymity, multicast and indirect communication features. In this architecture, publishers and subscribers do not need to share keys, so there is no burden of key management. Moreover, the architecture is defined on homomorphic encryption and supports intranetwork integration of encrypted data. This paper also analyzes the correctness and security of the proposed architecture. Preliminary estimates indicate that the implementation of the security section of this article proposed based publish / subscribe access control architecture has a good performance, and provides strong security capabilities (authorization and data confidentiality).

\section{Conclusions}

With the increasing opening and wide application of loT systems, security and privacy protection issues in the collaborative interaction of loT services are facing new challenges. This article proposes a secure data sharing mechanism for data sharing, service collaboration, combination, and user privacy in the Internet of Things environment. The I/O service interactive access control mechanism based on the publish/subscribe system protects data. Implement service privacy and access control. Aiming at the communication between large-scale collaborative services, this paper constructs an access control architecture suitable for the collaborative interaction of large-scale distributed Internet of Things service collaborative communication facilities and management services based on the publish/subscribe paradigm, and realizes the confidential service of data exchange. By randomly assigning different filters to different carrier signals to reduce the correlation of filter time-domain sampling values, the performance of the Internet of Things is optimized. This algorithm provides new ideas for solving loT security services without introducing additional computational complexity. Based on the communication facilities, this article has done two aspects of work. On the one hand, a data-centric access control architecture is proposed to implement access control to service sessions and protect the confidentiality of interactive data. The main idea of DCACF is to attach an access control strategy to the event, so that the event becomes an independent and meaningful entity. This architecture not only supports extensible smart grid service interaction, but also maintains service indirect, anonymous, multicast interaction characteristics and data confidentiality. Fully homomorphic encryption technology is used to realize the fusion of encrypted data within the grid.

\section{Declarations}




\section{Conflicts of interest}

The author of this article has no conflict of interest

\section{Availability of data and material}

No data were used to support this study.

\section{Authors' contributions}

Yuanguo Wang, Xiaogang Jiang: Writing and editing

Qian Yu: data analysis

Xiuling Zhang: Methodology

Bailu Zhao: Visualization

Xiaobei Wang: Software

Tonghui Han: Supervision

\section{Acknowledgements}

No grant was received for this article

\section{References}

1. Islam, N., Islam, N., \& Botnets (2017). and loT Security. Computer, 50(2), 76-79

2. Fernandes, E., Rahmati, A., Eykholt, K., et al. (2017). loT Security Research: A Rehash of Old Ideas or New Intellectual Challenges? IEEE Security \& Privacy, 15(4), 79-84

3. Maple, C. (2017). Security and privacy in the loT. Journal of Cyber Policy, 2(2), 155-184

4. Anitha, A. (2017). Home security system using loT. IOP Conference Series: Materials Science and Engineering, 263:042026.

5. Kolias, C., Meng, W., Kambourakis, G., et al. (2019). Security, Privacy, and Trust on loT. Wireless Communications and Mobile Computing, 2019, 1-3

6. Younis, M., Akkaya, K., \& Youssef, M. (2015). Handling QoS Traffic in Wireless Sensor Networks. IEEE Journal on Selected Areas in Communications, 28(7), 1105-1115

7. Kapileswar, N., \& Hancke, G. P. (2016). A Survey on Urban Traffic Management System Using Wireless Sensor Networks:. Sensors, 16(2), 157

8. Ndiaye, M., Hancke, G. P., \& Abu-Mahfouz, A. M. (2017). Software Defined Networking for Improved Wireless Sensor Network Management: A Survey. Sensors, 17(5), 1031 
9. Chen, Z., Liu, A., Li, Z., et al. (2016). Distributed duty cycle control for delay improvement in wireless sensor networks. Peer-to-Peer Networking and Applications, 10(3), 1-20

10. Malaver, A., Motta, N., Corke, P., et al. (2015). Development and Integration of a Solar Powered Unmanned Aerial Vehicle and a Wireless Sensor Network to Monitor Greenhouse Gases. Sensors, 15(2), 4072-4096

11. Khan, I., Belqasmi, F., Glitho, R., et al. (2017). Wireless sensor network virtualization: A survey. IEEE Communications Surveys \& Tutorials, 18(1), 553-576

12. Rashid, B., \& Rehmani, M. H. (2016). Applications of wireless sensor networks for urban areas: A survey. Journal of Network \& Computer Applications, 60, 192-219

13. Liu, X. Y., Zhu, Y., Kong, L., et al. (2015). CDC: Compressive Data Collection for Wireless Sensor Networks. IEEE Transactions on Parallel \& Distributed Systems, 26(8), 2188-2197

14. Srbinovska, M., Gavrovski, C., Dimcev, V., et al. (2015). Environmental parameters monitoring in precision agriculture using wireless sensor networks. Journal of Cleaner Production, 88, 297-307

15. Rezvani, M., Ignjatovic, A., Bertino, E., et al. (2015). Secure Data Aggregation Technique for Wireless Sensor Networks in the Presence of Collusion Attacks. Dependable \& Secure Computing IEEE Transactions on, 12(1), 98-110

\section{Figures}




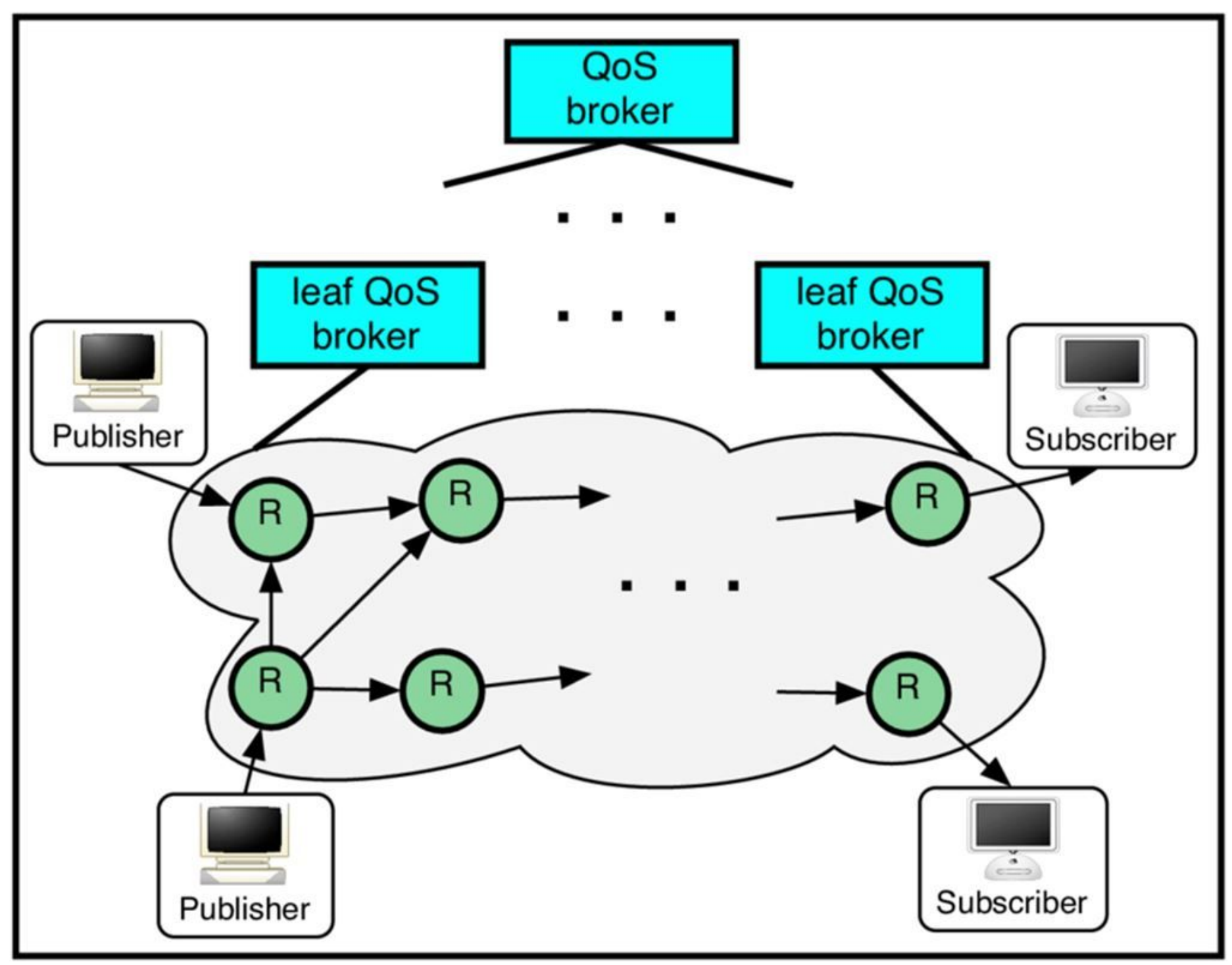

Figure 1

Grid Stat communication facility 


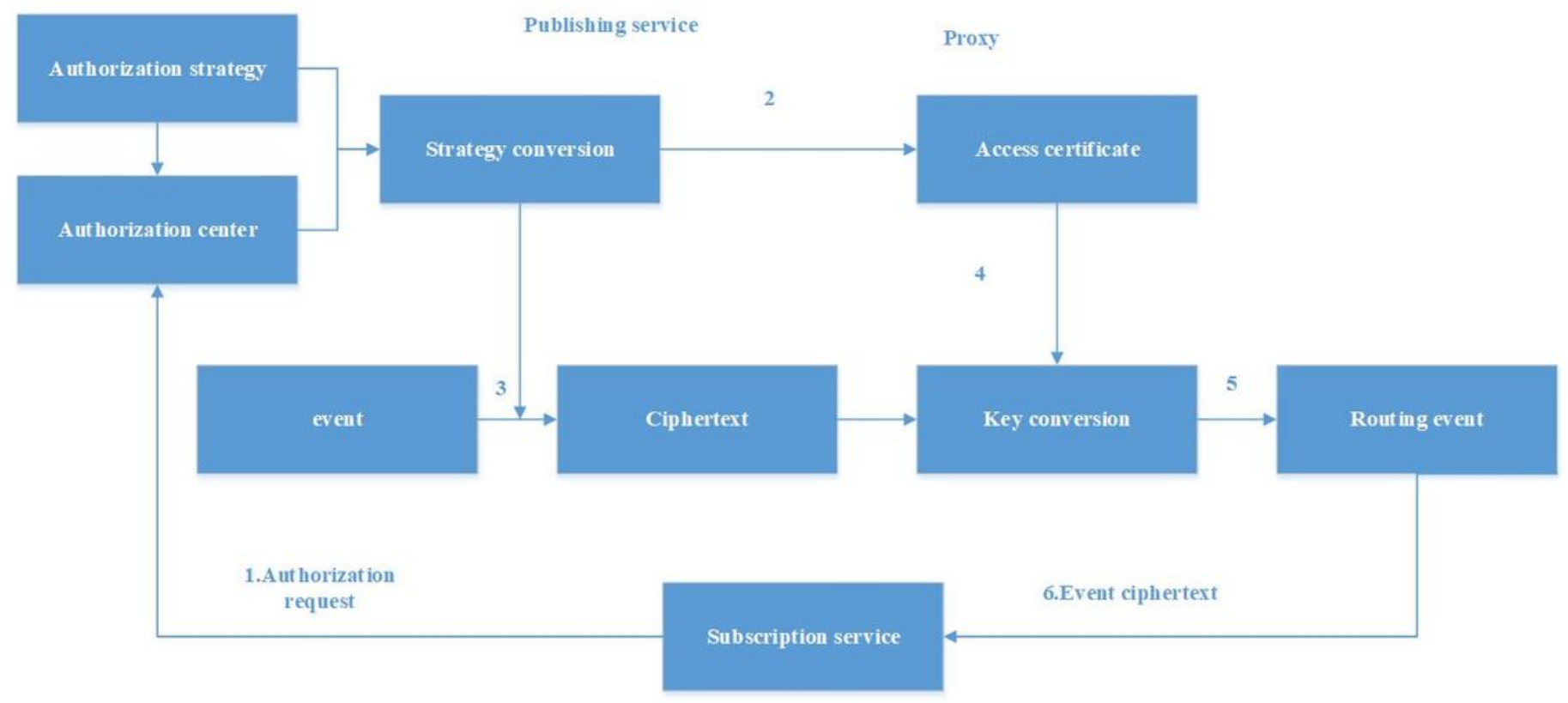

Figure 2

DCACF architecture 


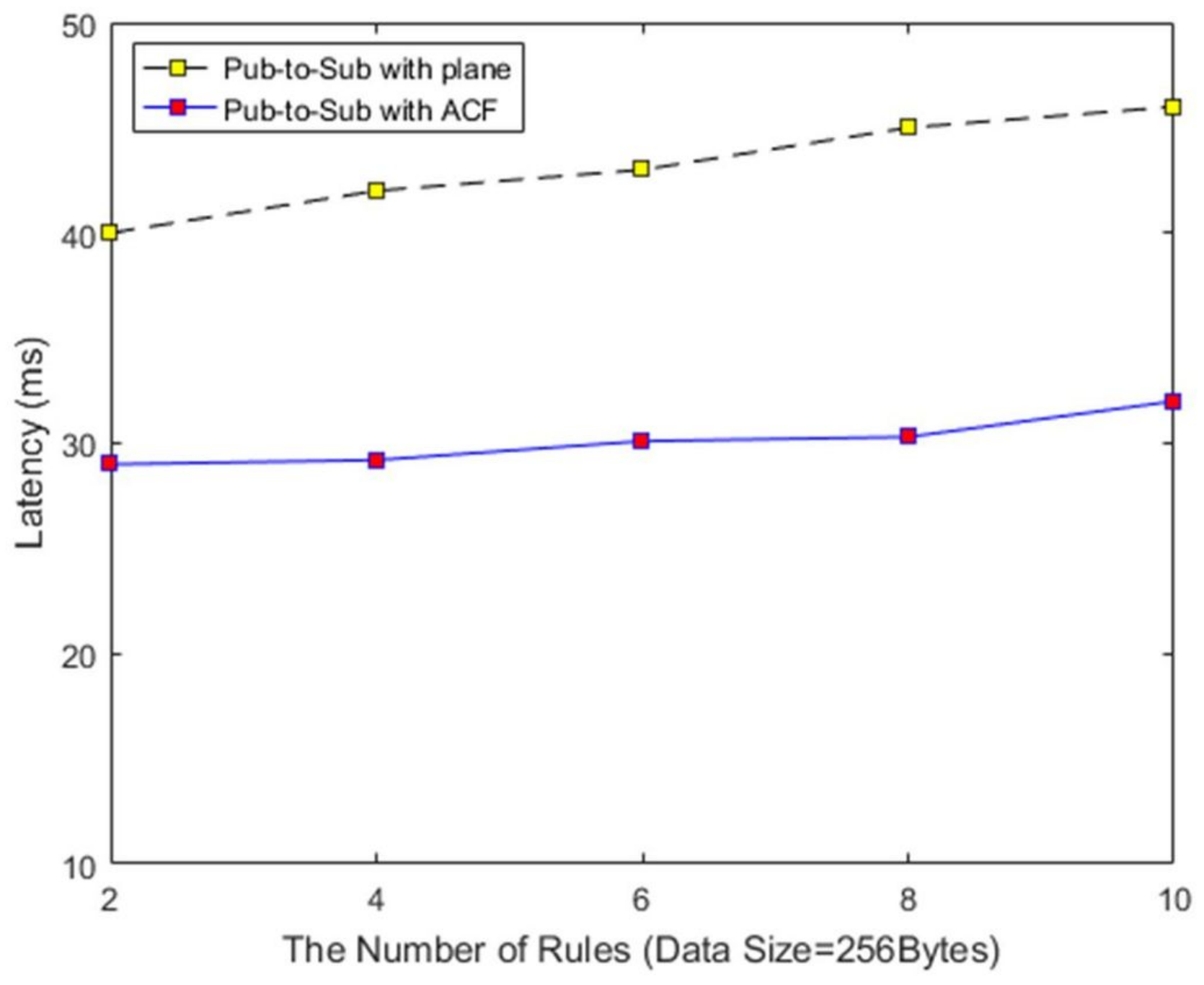

Figure 3

The number of data blocks affects the delay 


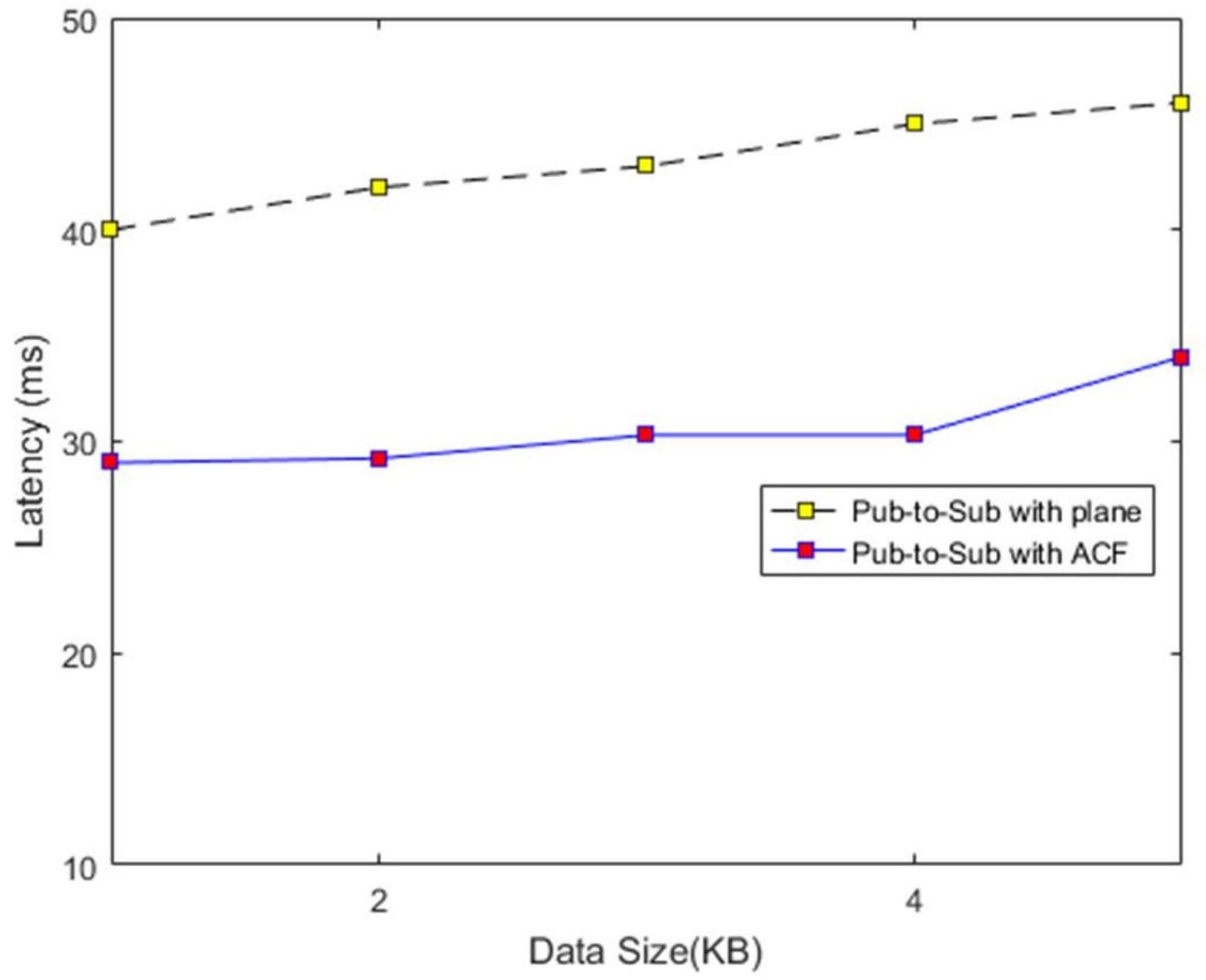

Figure 4

Effect of packet size on delay 